\title{
Impacto del uso de la Plataforma Virtual Moodle en el proceso de enseñanza aprendizaje en la Universidad Continental
}

\author{
Impact of the usage of the Moodle Virtual Platform in the teaching and \\ learning process in the Continental University
}

\author{
Carlos Peralta Santa Cruz', Carlos Calderon Sedano², Carol Rojas Moreno ${ }^{3}$ \\ Universidad Continental
}

\section{RESUMEN}

Objetivos: Determinar el impacto del uso de la plataforma virtual Moodle en el proceso de enseñanza aprendizaje de los estudiantes de pregrado de todas las carreras profesionales de la Universidad Continental. Métodos: La investigación es de tipo exploratorio, descriptivo. La población está conformada por todos los estudiantes matriculados en el semestre 2011 -I de pregrado que corresponde a 6370 estudiantes de las distintas carreras profesionales de la Universidad Continental; la muestra fue no probabilística, considerando a 362 estudiantes. Resultados: El conocimiento que tienen los estudiantes respecto al uso de la plataforma virtual Moodle en la Universidad Continental por parte de los estudiantes es de 87\%; los estudiantes de la facultad de Ingeniería en un $85 \%$ manifiestan que conocen la obligatoriedad de su uso y en la facultad de Ciencias de la Empresa sólo el $89 \%$. Respecto a la frecuencia de uso de la plataforma virtual Moodle, el $34 \%$ de estudiantes de la facultad de Ingeniería hace uso diario y en la facultad de Ciencias de la Empresa el $36 \%$ de estudiantes. El recurso más utilizado por los estudiantes es la descarga de archivos, que generalmente se trata de material de clase que los docentes publican; luego, el recurso menos utilizado es el chat. Conclusiones: Los estudiantes utilizan la plataforma virtual Moodle en su mayoría para realizar transacciones de descarga de contenidos, es decir como servidores de archivos, luego el uso de los recursos propios de la plataforma virtual diseñados para generar aprendizajes como foros,

chats, etc, son de poco uso.

Palabras Claves: Plataforma virtual, Moodle, enseñanza, aprendizaje.

1 Lic. en Matemática, candidato a Magister en Administración, Docente investigador de la Universidad Continental.

2 lng. de Computación y Sistemas, candidato a Magister en Administración, Docente investigador de la Universidad Continental.

3 lng. de Computación y Sistemas, candidata a Magister en Administración, Docente investigadora de la Universidad Continental. 


\section{ABSTRACT}

Objectives: To determine the impact of the usage of the Moodle Virtual Platform in the teaching and learning process of all professional careers undergraduate students of the Continental University. Methods: The research is of explorative and descriptive type. The population is formed by all undergraduate students enrolled in the 2011 -I semester, that corresponds to 6370 students of the diverse professionals careers of the Continental University; a non probability sampling considering 362 students. Results: The knowledge that the students have about usage of the Moodle Virtual Platform in the Continental University is of $87 \% ; 85 \%$ of students of the engeneering faculty demostrate that they know the obligatory of its use; and only $89 \%$ of business science faculty students. Respect to the usage frequency of the Moodle Virtual Platform, $34 \%$ of students from the engineering faculty makes daily use and in the business sciences faculty, 36\% of students. The most used resource by students is the files download, which generally is about the class material that professors publish; then, the less used resource in the chat. Conclusions: Students use the Moodle Virtual Platform in its majority to make contents download transactions, such as file servers, then the use of the own resources of the Moodle Virtual Platform, designed to generate learning such as forums, chats, etc, they are of a little use.

Key words: Moodle Virtual Platform, teaching, learning.

\section{INTRODUCCIÓN}

En el Perú existen investigaciones previas que manifiestan ya experiencias en el uso de plataformas virtuales.

Según Chunga, el uso de la Plataforma Virtual en los profesores de la Facultad de Humanidades de la Universidad Católica
Santo Toribio de Mogrovejo de la ciudad de Chiclayo, Perú, durante el ciclo académico $2008-1 I$, fue de un $96 \%$, sin embargo el $4 \%$ no utilizó esta plataforma. Complementariamente a estos resultados, los profesores plasmaron sus definiciones acertadas sobre una plataforma virtual. Además el $32 \%$ señaló haber utilizado otras plataformas, en contraposición al $68 \%$ que no ha utilizado otra plataforma. Las herramientas más valoradas como "muy buenas" en la plataforma virtual USAT fueron la opción: Documentos $(41,5 \%)$, tareas $(42,10 \%)$, foros $(32,4 \%)$. Contrario a ello, las herramientas más deficientes o limitadas son: el chat (50\%), Cuestionarios $(47,1 \%)$. Con ello, se afirma que la Plataforma Virtual por el momento se viene utilizando como espacio de repositorio de documentos online, complemento del desarrollo de asignaturas. (1)

Ejarque $E$, Buendía F, Hervás A, manifiestan que uno de los principales objetivos del campus virtual es conseguir mayor participación y mejorar la actitud del alumno ante la asignatura. Otro es motivar y formar al profesorado para que, poco a poco, vaya adaptando sus contenidos, prácticas, ejercicios y demás materiales al nuevo entorno, según las acciones propuestas. Tanto por el lado docente como por el del alumno debe fomentarse el intercambio de ideas, la comunicación, los debates, etc. Todo ello ayudado por herramientas de comunicación síncronas o asíncronas. Para conseguir el éxito en la implantación de un campus virtual, este debe realizarse de forma progresiva, con gran soporte institucional y un apoyo constante al colectivo docente para su motivación y formación en estas nuevas herramientas. Además debe existir una metodología, o unas guías a seguir a la hora de crear el entorno apropiado para el aprendizaje virtual de una asignatura (estructura de materiales, comunicación profesor alumno, comunicación alumno -alumno, etc.) tal como se ha presentado en este trabajo. (2)

Según Páez H. y Arreaza E. la experiencia realizada con la aplicación de la plataforma 
nicenet.org en las actividades de enseñanza aprendizaje de la asignatura Tecnología de la Información en Educación Superior constituye una reafirmación de las bondades de la incorporación de las tecnologías de la información y comunicación en educación. Las dificultades que encierran la diversidad en la localización espacial de los participantes, sus diferencias individuales en cuanto a la disponibilidad de tiempo útil de dedicación a los estudios fueron superadas con el uso de esta plataforma permitiendo a todos los participantes el logro de los objetivos del curso, el cual era elaborar un material educativo, con apoyo informático y sustento didáctico. (3)

Determinar el impacto del uso de la Plataforma Virtual Moodle en el proceso de enseñanza - aprendizaje de los estudiantes de pregrado de todas las carreras profesionales de la Universidad Continental.

Al familiarizarse con la plataforma, los participantes consignaron documentos, interactuaron masivamente en los trece foros de discusión planteados, revisaron los vínculos a web sites y colocaron algunos de éstos; además, cumplieron con los requisitos de participación y de evaluación establecidos. De igual manera, se observó la integración de los participantes en equipos y no en grupos de trabajo, beneficiándose de las fortalezas y superando las debilidades individuales, demostrando la ejecución de un trabajo colaborativo.

Así mismo, el trabajo colaborativo se incrementó a medida que se desarrollaban las actividades del curso como también la significación de los aprendizajes logrados por los participantes, dados el interés, motivación, relevancia e importancia de los materiales de consulta obtenidos a través de la red de redes para la realización de las investigaciones que debían realizar en el curso, y de la discusión de los mismos mediante la opción Conferencing de la plataforma y en las sesiones presenciales. Esta interacción favoreció el desarrollo de las habilidades de los participantes para discernir sobre la pertinencia de un material para la consecución de un objetivo. Además, esta experiencia permitió a los participantes mejorar o adquirir habilidades en el uso y manejo del computador por lo que se considera recomendable replicarla en sucesivos cursos (4).

Como se observa, en la actualidad, somos testigos de grandes avances en la tecnología y en la comunicación, diversos campos se han acogido a la nueva tecnología, debido a la facilidad y rapidez con que se puede manejar gran cantidad de información. Uno de los campos que han aprovechado la tecnología es el de la educación.

La educación Virtual persigue el aprendizaje sin que se produzca una coincidencia entre estudiante y docente ni en el espacio ni en el tiempo y asumen las funciones de contexto de aprendizaje que el aula desarrolla en el entorno presencial. Este enfoque de educación necesita de nuevos modelos pedagógicos para realizar las clases.

Investigaciones previas determinan que la Plataforma Moodle es considerada útil, práctica, fácil de usar y permite que tanto los docentes como los estudiantes puedan tener acceso a la misma información y que ésta quede disponible en el sistema, sin peligro de que se borre.

Se identifica las necesidades demandadas por los estudiantes universitarios en la actualidad frente a la tecnología existente y el uso de internet que cada vez es más común entre los jóvenes universitarios.

El problema que se ha planteado es ¿̇Cuál es el impacto del Uso de la Plataforma Virtual Moodle en el proceso de Enseñanza Aprendizaje en la Universidad Continental de Ciencias e Ingeniería?

El objetivo de este estudio es determinar el impacto del uso de la Plataforma Virtual Moodle en el proceso de enseñanza aprendizaje de los estudiantes de pregrado de todas las carreras profesionales de la Universidad Continenta.

En la Universidad Continental se utiliza la plataforma virtual Moodle que se encuentra 
a disposición de los docentes y alumnos para facilitar el proceso de enseñanza aprendizaje durante el semestre académico. Siendo la Universidad Continental una universidad líder en el uso de la tecnología en la región central de país.

\section{MATERIAL Y MÉTODOS}

La investigación es de tipo Exploratorio Descriptivo. La población está conformada por todos los estudiantes matriculados en el semestre 2011-I de pregrado que corresponde a 6370 estudiantes de las distintas carreras profesionales de la Universidad Continental; la muestra fue de 362 estudiantes, los cuales fueron distribuidos según la Tabla $N^{\circ} 1$.

El instrumento aplicado estuvo basado en un cuestionario de 33 preguntas de alternativa múltiple, con el cual se identifica el impacto del uso de la plataforma virtual.

Tabla № 1: Distribución de la población y tamaño muestra
La aplicación del instrumento fue a los estudiantes de las carreras profesionales que se indica en la Tabla $N^{\circ} 1$ en las aulas de clases y distribuidas aleatoriamente a una cantidad de estudiantes de acuerdo al tamaño muestral por carreras. Posteriormente se procesaron los resultados aplicando la estadística descriptiva para finalmente analizar el comportamiento de las variables objeto de estudio.

\section{RESULTADOS}

En cuanto al uso de la plataforma virtual por parte de los estudiantes, se observa que 362 alumnos opinaron que utilizan el aula virtual para descargar archivos de material de clases que el docente publica y 65 alumnos utilizan el chat para comunicarse con el docente y compañeros de clases (Tabla $N^{\circ} 2$ )

En relación a la interfaz que es utilizada por la plataforma del aula virtual se obtuvo que

\begin{tabular}{lcc}
\hline \multicolumn{1}{c}{ Escuela Académico Profesional } & Total & Muestra \\
\hline Administración: Marketing y Negocios Internacionales & 1369 & 78 \\
Arquitectura & 364 & 21 \\
Ciencias y Tecnologías de la Comunicación & 114 & 7 \\
Contabilidad & 579 & 33 \\
Derecho & 338 & 19 \\
Economía & 141 & 8 \\
Ingeniería Ambiental & 584 & 33 \\
Ingeniería Civil & 1046 & 60 \\
Ingeniería de Minas & 187 & 11 \\
Ingeniería de Sistemas e Informática & 657 & 37 \\
Ingeniería Eléctrica & 124 & 7 \\
Ingeniería en Agronegocios & 70 & 4 \\
Ingeniería Industrial & 336 & 19 \\
Ingeniería Mecánica & 70 & 4 \\
Psicología & 391 & 21 \\
Total & 6370 & 362 \\
\hline
\end{tabular}


Tabla № 2: Incidencias sobre los recursos que utilizan los alumnos en el aula virtual.

\begin{tabular}{|c|c|c|c|c|c|}
\hline \multirow{2}{*}{ Herramientas } & \multicolumn{2}{|c|}{$\begin{array}{l}\text { Facultad } \\
\text { Ingeniería }\end{array}$} & \multicolumn{2}{|c|}{$\begin{array}{l}\text { Facultad } \\
\text { Cs. de la Empresa }\end{array}$} & \multirow{2}{*}{ Tota } \\
\hline & $M$ & $F$ & $M$ & $F$ & \\
\hline $\begin{array}{l}\text { Para descargar archivos de material de } \\
\text { clase que el docente pública }\end{array}$ & 120 & 79 & 89 & 74 & 362 \\
\hline $\begin{array}{l}\text { Para descargar archivos de material de } \\
\text { apoyo a la clase que el docente pública }\end{array}$ & 129 & 75 & 91 & 66 & 361 \\
\hline $\begin{array}{l}\text { Para enviar archivos de trabajos al } \\
\text { docente }\end{array}$ & 120 & 93 & 86 & 59 & 358 \\
\hline $\begin{array}{l}\text { Para enviar archivos de exámenes } \\
\text { al docente }\end{array}$ & 100 & 79 & 91 & 62 & 332 \\
\hline $\begin{array}{l}\text { Para rendir evaluaciones, tipo } \\
\text { cuestionario }\end{array}$ & 97 & 65 & 88 & 51 & 301 \\
\hline $\begin{array}{l}\text { Para dar opinión en el foro publicado } \\
\text { por el docente }\end{array}$ & 80 & 71 & 51 & 42 & 244 \\
\hline $\begin{array}{l}\text { Para enviar mensajes de texto, para } \\
\text { consultar al docente }\end{array}$ & 47 & 47 & 37 & 37 & 168 \\
\hline $\begin{array}{l}\text { Para enviar mensajes de texto a tus } \\
\text { compañeros }\end{array}$ & 31 & 17 & 34 & 28 & 110 \\
\hline $\begin{array}{l}\text { Para realizar conversaciones en línea } \\
\text { (chat) con el docente }\end{array}$ & 19 & 15 & 20 & 11 & 65 \\
\hline $\begin{array}{l}\text { Para realizar conversaciones en línea } \\
\text { (chat) con sus compañeros }\end{array}$ & 5 & 1 & 4 & 3 & 13 \\
\hline
\end{tabular}

el $39 \%$ de los alumnos de la Facultad de ingeniería manifiestan que los colores de la interfaz son adecuados, en cambio un $28 \%$ opina que los recursos están adecuadamente organizados para un fácil uso; por otro lado los estudiantes de la Facultad de Ciencias de la Empresa en un $36 \%$ manifestaron que los colores de la interfaz son adecuados, y un $28 \%$ opinan que los recursos están adecuadamente organizados para un fácil uso.

Respecto al uso del aula virtual como un apoyo académico utilizado por los docentes, se obtuvo un $45 \%$ de los alumnos de la Facultad de Ingeniería manifiestan que todos los docentes utilizan el aula virtual, un $32 \%$ opinan que algunos docentes usan el aula virtual como apoyo académico. En cambio un $46 \%$ de los alumnos de la Facultad de Ciencias de la Empresa manifiestan que los docentes utilizan el aula virtual, y un $36 \%$ opinan que todos los docentes usan el aula virtual como apoyo académico.
Sobre el momento de aprendizaje del uso de la plataforma virtual Moodle, el $71 \%$ de los estudiantes han aprendido el uso de la plataforma virtual en el primer ciclo en la asignatura de Informática I en la Facultad de Ingeniería, y el $73 \%$ en la Facultad de Ciencias de la Empresa.

Cuando se revisó la frecuencia de uso de la plataforma virtual Moodle el $34 \%$ de estudiantes de la Facultad de Ingeniería hace uso diario de la plataforma y el $36 \%$ de la Facultad de Ciencias de la Empresa.

Respecto al acceso a la plataforma virtual Moodle, el 58\% logra el acceso con facilidad en la Facultad de Ingeniería y el $65 \%$ en la Facultad de Ciencias de la Empresa. 


\section{DISCUSIÓN}

De acuerdo a Chunga, el uso de la Plataforma Virtual en los profesores de la Facultad de Humanidades de la Universidad Católica Santo Toribio de Mogrovejo fue de un $96 \%$, sin embargo el $4 \%$ no utilizó esta plataforma. Las herramientas más valoradas como "muy buenas" en la plataforma virtual USAT fueron la opción: Documentos $(41,5 \%)$, tareas $(42,10 \%)$, foros $(32,4 \%)$. Contrario a ello, las herramientas más deficientes o limitadas son: el chat (50\%), Cuestionarios (47,1\%). Con ello, se afirma que la Plataforma Virtual por el momento se viene utilizando como espacio de repositorio de documentos online, complemento del desarrollo de asignaturas. (1) En nuestro estudio se ha determinado que el conocimiento sobre la obligatoriedad del uso de la plataforma virtual Moodle por parte de los estudiantes de la Universidad Continental es del $87 \%$. Los estudiantes utilizan la plataforma virtual Moodle para descargar archivos del material de clase que el docente publica, siendo el recurso menos utilizado el servicio chat. Asimismo, los estudiantes manifiestan que los docentes en su mayoría sólo utilizan la plataforma virtual Moodle para publicar archivos de apoyo a la clase y que no utilizan el chat para comunicarse con los alumnos. De esta forma se demuestra que en ambos estudios existe coincidencia respecto a los usos de la plataforma virtual.

En conclusión, los estudiantes utilizan la plataforma virtual Moodle en su mayoría para realizar transacciones de descarga de contenidos que son publicados por los docentes, es decir como servidores de archivos, luego el uso de los recursos propios de la plataforma virtual diseñados para generar aprendizajes como foros, chats, etc, son de poco uso. 


\section{REFERENCIAS BIBLIOGRAFICAS}

1. Chunga G. Uso de una Plataforma Virtual en los profesores de una Facultad de Humanidades [Internet]. 2008. Consultado el 8 de enero de 2011. Disponible en: http://www.usat.edu.pe/usat/ facultad-humanidades/files/2011/05/uso-de-unaplataforma-virtual-en-los-profesores-de-una-facultad-de-humanidades.pdf

2. Ejarque E, Buendía F, Hervás A. Estudio sobre el impacto del uso de un campus virtual en la enseñanza universitaria [Internet]. Consultado el 11 de febrero de 2011. Disponible en: http://ddd.vab.cat/pub/educar/0211819Xn41p11.pdf.

3. Páez H, Arreaza E. Percepción del aprendizaje combinado en la educación de postgrado, visión del participante [Internet]. Carabobo: Universidad de Carabobo, Venezuela; 2007. Disponible en: http://www.ucv.ve/fileadmin/user_upload/vrac/ documentos/Curricular_Documentos/Evento/Ponencias/Arreaza_Evelyn_y_otros.pdf

4. Arteaga R. Análisis de las plataformas de enseñanza virtuales, desde la perspectiva del TAM [Internet]. Consultado el 14 de enero de 2011 . Disponible en: http://www.gabinetecomunicacionyeducacion.com/files/adjuntos/An\%C3\%Al lisis\%20de\% 20las\%20plataformas\%20de\%20ense\%C3\%B 1 anza\%20virtuales\%20desde\%20la\%20pers pectiva\%20del\%20TAM.pdf

Correo electrónico:

ccalderon@continental.edu.pe cperalta@continental.edu.pe

crojas@continental.edu.pe 